\title{
On bicomplex numbers with coefficients from the complex Fibonacci sequence
}

\author{
Serpil Halıcı ${ }^{1}$ and Şule Çürüik ${ }^{2}$ \\ ${ }^{1}$ Department of Mathematics, Faculty of Sciences and Arts \\ University of Pamukkale, Turkey \\ e-mail: shaliciepau.edu.tr \\ ${ }^{2}$ Department of Mathematics, Faculty of Sciences and Arts \\ University of Pamukkale, Turkey \\ e-mail: scuruk10@posta.pau.edu.tr
}

Received: 18 January 2019

Revised: 15 July 2019

Accepted: 20 July 2019

\begin{abstract}
The aim of this paper is to introduce a new sequence of bicomplex numbers with coefficients from the complex Fibonacci sequence, and to investigate some fundamental properties of the newly defined sequence.
\end{abstract}

Keywords: Bicomplex number, Fibonacci sequence.

2010 Mathematics Subject Classification: 11B39, 11R52.

\section{Introduction}

Quaternions are the noncommutative normed division algebra over the real numbers and they are represented in the form of hyper-complex numbers with three imaginary components;

$$
q=\left(a_{0}, a_{1}, a_{2}, a_{3}\right)=a_{0}+a_{1} i+a_{2} j+a_{3} i j,
$$

where $i, j$ and $i j=k$ are mutually perpendicular unit bivectors and $a_{0}, a_{1}, a_{2}, a_{3}$ are real numbers [8]. These numbers obey the famous multiplication rules discovered by Hamilton in 1843; $i^{2}=j^{2}=k^{2}=i j k=-1$. Hamilton quaternions form a division algebra. Later, in 1892, Segre defined bicomplex numbers [18] which used to be neglected because of their zero 
divisors, but now they are attracting the researchers' attention. Since the mathematical structure of quantum mechanics is studied on the complex number field, there are many authors working on bicomplex numbers in this area (see, [1,11,13-17]). Also, some researchers have studied algebraic, geometric, topological and dynamic properties of bicomplex numbers (see, [11,16,17]).

The set of bicomplex numbers $\mathbb{B} \mathbb{C}$, is defined as follows [14]:

$$
\mathbb{B C}=\left\{z_{1}+z_{2} j \mid z_{1}, z_{2} \in \mathbb{B} \mathbb{C}, j^{2}=-1\right\}
$$

Any element $b$ of $\mathbb{B} \mathbb{C}$ is actually written, for $t=1,2,3,4$ and $a_{t} \in \mathbb{R}$, as $b=a_{1}+a_{2} i+a_{3} j+a_{4} i j$. The element $b=1$ is the unit element. And the following equalities are known among other basis elements $\{1, i, j, k\}$ :

$$
i j=j i=k, i k=k i=-j, j k=k j=-i
$$

and $i^{2}=j^{2}=-1,(i j)^{2}=1$, where $i, j$ and $i j=k$ are imaginary and hyperbolic units, respectively. Two bicomplex numbers are equal if all their components are equal, one by one. The sum of two bicomplex numbers is defined by summing their components. The addition operation in the set of bicomplex numbers is both commutative and associative. Zero is the null element, and the inverse of the element $b$ with respect to the addition is $-b$ which is defined as having all the coefficients of $b$ changed in their signs. This implies that $(\mathbb{B C},+)$ is an Abelian group. The bicomplex product is obtained by taking into account the multiplication of the base elements $\{1, i, j, k\}$.

In $\mathbb{B C}$, using imaginary and hyperbolic units, three different conjugate definitions can also be given as $\overline{b_{i}}=a_{1}-a_{2} i+a_{3} j-a_{4} k, \overline{b_{j}}=a_{1}+a_{2} i-a_{3} j-a_{4} k$ and $\overline{b_{i j}}=a_{1}-a_{2} i-a_{3} j+a_{4} k$.

Due to the different conjugates, there are three different norm definitions as

$$
\begin{gathered}
|b|_{i}^{2}=b \overline{b_{i}}=\left(\left|z_{1}\right|^{2}-\left|z_{2}\right|^{2}\right)+2 \operatorname{Re}\left(z_{1} \overline{z_{2}}\right) j, \\
|b|_{i j}^{2}=b \overline{b_{i j}}=\left|z_{1}\right|^{2}+\left|z_{2}\right|^{2}-2 \operatorname{Im}\left(z_{1} \overline{z_{2}}\right) k,
\end{gathered}
$$

and

$$
|b|_{j}^{2}=b \overline{b_{j}}=z_{1}^{2}+z_{2}^{2} .
$$

In this work, we remind some properties of bicomplex numbers by examining the conjugates and norms. Then, we introduce the set of bicomplex numbers with coefficient in complex Fibonacci sequence, and give some fundamental properties of the defined numbers. Also, we obtain some generalized identities associated with this sequence, such as Catalan, d'Ocagne, Honsberger, etc. identities.

\section{Bicomplex numbers whose coefficients are from the complex Fibonacci sequence}

As it is well-known the $n$-th Fibonacci and Lucas quaternions were defined by Horadam, for $n \geq 0$,

$$
Q_{n}=F_{n}+i F_{n+1}+j F_{n+2}+k F_{n+3}
$$


and

$$
K_{n}=L_{n}+i L_{n+1}+j L_{n+2}+k L_{n+3},
$$

respectively $[9,10]$. In [6], Halicihave defined complex Fibonacci quaternions and gave some important and basic properties. Then, in [4], the authors have considered quaternion and symbol algebras and studied their properties. In [15], Nurkan and Guven defined the $n$-th bicomplex Fibonacci and Lucas numbers using the basis elements of bicomplex numbers, as follows:

$$
B F_{n}=F_{n}+i F_{n+1}+j F_{n+2}+k F_{n+3}
$$

and

$$
B L_{n}=L_{n}+i L_{n+1}+j L_{n+2}+k L_{n+3},
$$

respectively, where $F_{n}$ and $L_{n}$ are the $n$-th Fibonacci and Lucas numbers. The authors gave Binet's formula involving these numbers. Also, they obtained some important identities and relations involving these numbers. A similar sequence has been studied in $[2,3]$ by Deveci and Shannon. And then, in [7], bicomplex Fibonacci numbers and a generalization of theirs has been studied. Motivated by these references, in this section we firstly examine bicomplex numbers whose coefficients are from the Fibonacci sequence.

Let us use $\mathbb{B C}_{F}$ notation to represent the set of bicomplex numbers whose coefficients are from the Fibonacci sequence. That is, $\mathbb{B} \mathbb{C}_{F}$ is

$$
\mathbb{B C}_{F}=\left\{B F_{n} \mid B F_{n}=F_{n}+F_{n+1} i+F_{n+2} j+F_{n+3} k\right\},
$$

where $B F_{n}=F_{n}+i F_{n+1}+j F_{n+2}+k F_{n+3}$. The algebraic operations in the set $\mathbb{B} \mathbb{C}_{F}$ are as follows:

$$
\begin{gathered}
B F_{n} \pm B F_{m}=\left(F_{n} \pm F_{m}\right)+i\left(F_{n+1} \pm F_{m+1}\right)+j\left(F_{n+2} \pm F_{m+2}\right)+k\left(F_{n+3} \pm F_{m+3}\right), \\
B F_{n} B F_{m}=\left(F_{n} F_{m}-F_{n+1} F_{m+1}-F_{n+2} F_{m+2}+F_{n+3} F_{m+3}\right)+X i+Y j+Z k,
\end{gathered}
$$

where $X, Y, Z$ are

$$
\begin{aligned}
& X=F_{n} F_{m+1}+F_{n+1} F_{m}-F_{n+2} F_{m+3}-F_{n+3} F_{m+2}, \\
& Y=F_{n} F_{m+2}-F_{n+1} F_{m+3}+F_{n+2} F_{m}-F_{n+3} F_{m+1}, \\
& Z=F_{n} F_{m+3}+F_{n+1} F_{m+2}+F_{n+2} F_{m+1}+F_{n+3} F_{m} .
\end{aligned}
$$

The conjugates of the $n$-th Fibonacci bicomplex number, $B F_{n}$, with respect to $i, j, k$ units can be defined as follows:

$$
\begin{aligned}
& B F_{n}^{i}=F_{n}-F_{n+1} i+F_{n+2} j-F_{n+3} k ; i-\text { conjugate }, \\
& B F_{n}^{j}=F_{n}+F_{n+1} i-F_{n+2} j-F_{n+3} k ; j-\text { conjugate, } \\
& B F_{n}^{k}=F_{n}-F_{n+1} i-F_{n+2} j+F_{n+3} k ; k \text { - conjugate. }
\end{aligned}
$$

From the above definitions of conjugates, we get

$$
B F_{n}^{i}=F_{n} B F_{1}^{i}+F_{n-1} B F_{0}^{i}
$$




$$
\begin{aligned}
& B F_{n}^{j}=F_{n} B F_{1}^{j}+F_{n-1} B F_{0}^{j}, \\
& B F_{n}^{k}=F_{n} B F_{1}^{k}+F_{n-1} B F_{0}^{k},
\end{aligned}
$$

where $B F_{1}=1+i+2 j+3 k$ and $B F_{0}=i+j+2 k$. There are relationships among bicomplex numbers, their conjugates and Fibonacci numbers $F_{n}$. Let us give some of these relationships:

$$
\begin{aligned}
& \frac{1}{2}\left(B F_{n}+B F_{n}^{i}\right)=F_{n}+j F_{n+2}=(1+2 j) F_{n}+j F_{n-1}, \\
& \frac{1}{2}\left(B F_{n}+B F_{n}^{j}\right)=F_{n}+i F_{n+1}=(1+i) F_{n}+i F_{n-1}, \\
& \frac{1}{2}\left(B F_{n}+B F_{n}^{k}\right)=F_{n}+k F_{n+3}=(1+3 k) F_{n}+2 k F_{n-1}, \\
& \frac{1}{2}\left(B F_{n}^{i}+B F_{n}^{j}\right)=F_{n}-k F_{n+3}=(1-3 k) F_{n}-2 k F_{n-1}, \\
& \frac{1}{2}\left(B F_{n}^{i}+B F_{n}^{k}\right)=F_{n}-i F_{n+1}=(1-i) F_{n}-i F_{n-1}, \\
& \frac{1}{2}\left(B F_{n}^{j}+B F_{n}^{k}\right)=F_{n}-j F_{n+2}=(1-2 j) F_{n}-j F_{n-1} .
\end{aligned}
$$

Thus, by using the definitions of $B F_{n}^{i}, B F_{n}^{j}, B F_{n}^{k}$ we can calculate the norms of the elements $B F_{n}$ in $\mathbb{B C}_{F}$ :

$$
\begin{gathered}
\left(\left|B F_{n}\right|_{i}\right)^{2}=B F_{n} B F_{n}^{i}=-L_{2 n+3}+2 j F_{2 n+3}, \\
\left(\left|B F_{n}\right|_{j}\right)^{2}=-\left(F_{2 n+3}-2 F_{n+2} F_{n+1}\right)+2 i\left(F_{2 n+3}+2 F_{n} F_{n+1}\right), \\
\left(\left|B F_{n}\right|_{k}\right)^{2}=B F_{n} B F_{n}^{k}=3 F_{2 n+3}+2 k(-1)^{n+1} .
\end{gathered}
$$

In [9], Horadam defined the $n$-th complex Fibonacci number as

$$
C_{n}=F_{n}+i F_{n+1}
$$

where $i^{2}=-1$. Also, Horadam examined the quaternion recurrence relations [10]. In 2012, Halıcidefined the quaternions with components from Fibonacci numbers and gave some important fundamental properties of them [5].

In this study, following Nurkan [15] and Halıc1 [5], we define bicomplex numbers which have coefficients in complex Fibonacci numbers. We investigate some important properties of these numbers and gave their Binet formula. Then, we use the Binet's formula to show some important properties of the newly defined numbers. Also, we get the several well-known generalized identities related to these numbers.

Let us define $n$-th bicomplex number, which has its coefficients from the complex Fibonacci numbers, as follows:

$$
B_{n}=C_{n}+C_{n+1} i+C_{n+2} j+C_{n+3} k,
$$

where $n$ is an integer, $C_{n}=F_{n}+i F_{n+1}$ and $\{1, i, j, k\}$ are the basis elements in $\mathbb{B C}$. By the aid of some elementary calculations, we find the following recursive relation for bicomplex numbers:

$$
B_{n}+B_{n+1}=C_{n+2}+i C_{n+3}+j C_{n+4}+k C_{n+5}=B_{n+2} .
$$


We denote the set of numbers $B_{n}$ by $\mathbb{B C}_{C F}$ :

$$
\mathbb{B C}_{C F}=\left\{B_{n} \mid B_{n}=C_{n}+C_{n+1} i+C_{n+2} j+C_{n+3} i j, i^{2}=j^{2}=-1, k^{2}=1\right\} .
$$

Note that any bicomplex number $B_{n}$ consists of scalar and vector parts:

$$
B_{n}=S_{B_{n}}+V_{B_{n}}=T_{0}+T
$$

where $T_{0}$ and $T$ are $T_{0}=C_{n}=F_{n}+i F_{n+1}, T=C_{n+1} i+C_{n+2} j+C_{n+3} k$, respectively. Using this representation for $B_{n}$, the addition and multiplication operations can be done more easily. Indeed, if we write $B_{n}=T_{0}+T$ and $B_{n+1}=T_{0}^{\prime}+T^{\prime}$, then we write

$$
\begin{gathered}
B_{n}+B_{n+1}=\left(T_{0}+T_{0}^{\prime}\right)+\left(T+T^{\prime}\right), \\
B_{n} B_{n+1}=T_{0} T_{0}^{\prime}+T_{0} T^{\prime}+T_{0}^{\prime} T-T \cdot T^{\prime}+T \times T^{\prime},
\end{gathered}
$$

where $($.$) and (x)$ denote the dot and vector product, respectively. According to the imaginary units three different norms can be given in the set $\mathbb{B C}_{C F}$ as follows:

$$
\begin{aligned}
& \text { i) } N B_{n}{ }^{i}=F_{n}^{2}-2 F_{n+2}^{2}+F_{n+4}^{2}+2\left(i a_{1}+j b_{1}-k c_{1}\right) \\
& \text { ii) } N B_{n}{ }^{j}=F_{n}^{2}-4 F_{n+1}^{2}+2 F_{n+2}^{2}-4 F_{n+3}^{2}+F_{n+4}^{2}-2 F_{n+2} F_{n+4}-4 i\left(F_{n+1}^{2}+F_{n+3}^{2}\right) \\
& \text { iii) } N B_{n}{ }^{k}=F_{n}^{2}-F_{n+4}^{2}+2\left(i a_{2}-j b_{2}+k c_{2}\right)
\end{aligned}
$$

where

$$
\begin{gathered}
a_{1}=\left(F_{n+1}^{2}-F_{n+2}^{2}-F_{n+2} F_{n+1}-F_{n+2} F_{n+3}-F_{n+3}^{2}\right), \\
c_{1}=F_{n} F_{n+3}+F_{n+1} F_{n+2}+F_{n+1} F_{n+4}+F_{n+2} F_{n+3}, \\
b_{1}=F_{n} F_{n+2}-F_{n+2} F_{n+4}, \\
a_{2}=3 F_{n}^{2}+12 F_{n} F_{n+1}+8 F_{n+1}^{2}, \\
c_{2}=F_{n} F_{n+3}-F_{n} F_{n+4}+F_{n+2}^{2}, \\
b_{2}=F_{n} F_{n+4}-F_{n+2}^{2} .
\end{gathered}
$$

Since $F_{n} \neq 0$, the above all norms cannot be zero. Thus, we get $i, j, k$ - inverses for the number $B_{n}$ as follows:

$$
\left(B_{n}^{i}\right)^{-1}=\frac{B_{n}^{i}}{N B_{n}{ }^{i}} ;\left(B_{n}^{j}\right)^{-1}=\frac{B_{n}^{j}}{N B_{n}{ }^{j}} ;\left(B_{n}^{k}\right)^{-1}=\frac{B_{n}^{k}}{N B_{n}{ }^{k}} .
$$

The following theorem gives Binet formula for the $n$-th bicomplex number whose coefficients are from the complex Fibonacci numbers.

Theorem 2.1 (Binet Formula). For $n \geq 0$, the formula giving the $n$-th number $B_{n}$ is

$$
B_{n}=B_{0} F_{n-1}+B_{1} F_{n}=\frac{1}{\sqrt{5}}\left(\underline{\alpha} \alpha^{n}+\underline{\beta} \beta^{n}\right),
$$

where

$$
\underline{\alpha}=\alpha(2 i-1)+\alpha^{3}(2 k-j), \underline{\beta}=\beta(1-2 i)+\beta^{3}(j-2 k) .
$$


Proof. The general term of the sequence $\mathbb{B C}_{C F}$ is

$$
B_{n}=A \alpha^{n}+B \beta^{n},
$$

where constants $A$ and $B$ are obtained by using the initial conditions as

$$
A=\frac{B_{1}-\beta B_{0}}{\sqrt{5}}, B=\frac{\alpha B_{0}-B_{1}}{\sqrt{5}} .
$$

If we substitute these values in the equation, then we get

$$
\begin{gathered}
B_{n}=\frac{B_{1} \alpha^{n}-\beta \alpha^{n} B_{0}+\alpha \beta^{n} B_{0}-B_{1} \beta^{n}}{\sqrt{5}}, \\
B_{n}=\frac{1}{\sqrt{5}}\left\{\left(B_{1}-\beta B_{0}\right) \alpha^{n}+\left(\alpha B_{0}-B_{1}\right) \beta^{n}\right\}, \\
B_{n}=\frac{1}{\sqrt{5}}\left(\underline{\alpha} \alpha^{n}+\underline{\beta} \beta^{n}\right),
\end{gathered}
$$

where

$$
\underline{\alpha}=\alpha(2 i-1)+\alpha^{3}(2 k-j), \underline{\beta}=\beta(1-2 i)+\beta^{3}(j-2 k) .
$$

Thus, the theorem is proved.

The generating function is equivalent to the Binet formula which helps to find any element. That is, the Binet formula can be controlled by the generating function.

The following theorem gives the generating function for the bicomplex number with coefficients from complex Fibonacci numbers.

Theorem 2.2 (Generating Function). For $B_{n}$, the generating function is

$$
G(t)=\frac{(-1+2 i+(2+t)(-j+2 k)}{\left(1-t-t^{2}\right)} .
$$

Proof. The generating function is

$$
G(t)=B_{0}+B_{1} t+B_{2} t^{2}+\ldots+B_{n} t^{n}+\ldots
$$

If we multiply this equation by $-t$ and $-t^{2}$, that is

$$
-t G(t)=-\left(B_{0} t+B_{1} t^{2}+B_{2} t^{3}+\ldots+B_{n} t^{n+1}+\ldots\right)
$$

and

$$
-t^{2} G(t)=-\left(B_{0} t^{2}+B_{1} t^{3}+B_{2} t^{4}+\ldots+B_{n} t^{n+2}+\ldots\right),
$$

then, making the necessary steps to find $G(t)$ with the help of the recurrence relation $B_{n}$, we get

$$
G(t)=\frac{B_{0}+\left(B_{1}-B_{0}\right) t}{1-t-t^{2}},
$$

where

$$
B_{0}=-1+2 i-2 j+4 k, B_{1}=-1+2 i-3 j+6 k
$$

are the initial values for $B_{n}$. Thus we find that

$$
G(t)=\frac{(-1+2 i+(2+t)(-j+2 k)}{\left(1-t-t^{2}\right)} .
$$

So, the proof is complete. 
Corollary 2.2.1. For the numbers $B_{n}, \overline{B_{n}}, B_{n}^{i}, B_{n}^{j}$ and $B_{n}^{k}$ the following equalities are satisfied:

$$
\begin{gathered}
\frac{1}{2}\left(B_{n}+\overline{B_{n}}\right)=Q_{n}, \\
\left(B_{n}^{i}+B_{n}^{j}+B_{n}^{k}\right)=3 C_{n}-i C_{n+1}-j C_{n+2}-k C_{n+3}, \\
\frac{1}{4}\left(B_{n}+B_{n}^{i}+B_{n}^{j}+B_{n}^{k}\right)=C_{n} .
\end{gathered}
$$

Corollary 2.2.2. For the negative integers $n, B_{-n}$ is as follows:

$$
B_{-n}=(-1)^{n}\left\{\left(F_{n-2}-F_{n}\right)+2 F_{n-1} i+\left(F_{n-4}-F_{n-2}\right) j+\left(F_{n+3}+F_{n-3}\right) k\right\} .
$$

Proof. Using the identity $F_{-n}=(-1)^{n+1} F_{n}$, we write

$$
\begin{aligned}
B_{-n}= & \left\{(-1)^{n+1} F_{n}+i(-1)^{n+2} F_{n-1}\right\}+\left\{(-1)^{n+2} F_{n-1}+i(-1)^{n+3} F_{n-2}\right\} i \\
& +\left\{(-1)^{n+3} F_{n-2}+i(-1)^{n+4} F_{n-3}\right\} j+\left\{(-1)^{n+4} F_{n-3}+i(-1)^{n+5} F_{n-4}\right\} k .
\end{aligned}
$$

Making the necessary arrangements, we get

$$
B_{-n}=(-1)^{n}\left(\left(-F_{n}+i F_{n-1}\right)+\left(F_{n-1}-i F_{n-2}\right) i+\left(-F_{n-2}+i F_{n+3}\right) j+\left(F_{n-3}-i F_{n-4}\right) k\right)
$$

which is the desired result.

Theorem 2.3 (Cassini's Identity). For the elements $B_{n}$ of the sequence $\mathbb{B C}_{C F}$, we have

$$
B_{n-1} B_{n+1}-B_{n}^{2}=6(-1)^{n-1}(3 j-4 k) .
$$

Proof. We prove this claim by direct calculation. For this purpose, using the Binet formula for the numbers $B_{n}$, we can write

$$
\begin{gathered}
B_{n-1} B_{n+1}-B_{n}^{2}=\frac{1}{\sqrt{5}}\left(\underline{\alpha} \alpha^{n-1}+\underline{\beta} \beta^{n-1}\right) \frac{1}{\sqrt{5}}\left(\underline{\alpha} \alpha^{n+1}+\underline{\beta} \beta^{n+1}\right)-\frac{1}{5}\left(\underline{\alpha} \alpha^{n}+\underline{\beta} \beta^{n}\right)^{2} \\
B_{n-1} B_{n+1}-B_{n}^{2}=\frac{1}{5}\left(\underline{\alpha} \alpha^{n-1} \underline{\beta} \beta^{n+1}+\underline{\beta} \beta^{n-1} \underline{\alpha} \alpha^{n+1}-2 \underline{\alpha} \alpha^{n} \underline{\beta} \beta^{n}\right) .
\end{gathered}
$$

Making the necessary arrangements and calculations, we obtain

$$
B_{n-1} B_{n+1}-B_{n}^{2}=(-1)^{n-1} \underline{\alpha} \underline{\beta} .
$$

Calculating the value $\underline{\alpha} \underline{\beta}$, we find that

$$
\underline{\alpha} \underline{\beta}=6(3 j-4 k) .
$$

Thus, the proof is completed.

Theorem 2.4 (Catalan's Identity). For the integers $n \geq k$ and the numbers $B_{n}$, the following equality is true:

$$
B_{n+k} B_{n-k}-B_{n}^{2}=\frac{6}{5}(-1)^{n}(3 j-4 k)\left\{(-1)^{k}\left(\alpha^{k}+\beta^{k}\right)^{2}-4\right\} .
$$


Proof. Let us use Binet formula for $B_{n}$ to prove this theorem. Hence, the formula $B_{n+k} B_{n-k}-B_{n}^{2}$ is equal to

$$
\frac{1}{\sqrt{5}}\left(\underline{\alpha} \alpha^{n+k}+\underline{\beta} \beta^{n+k}\right) \frac{1}{\sqrt{5}}\left(\underline{\alpha} \alpha^{n-k}+\underline{\beta} \beta^{n-k}\right)-\frac{1}{5}\left(\underline{\alpha} \alpha^{n}+\underline{\beta} \beta^{n}\right)^{2} .
$$

Thus, we obtain

$$
\frac{1}{5}\left(\underline{\alpha}^{2} \alpha^{2 n}+\underline{\alpha} \underline{\beta} \alpha^{n+k} \beta^{n-k}+\underline{\beta} \underline{\alpha} \beta^{n+k} \alpha^{n-k}+\underline{\beta}^{2} \beta^{2 n}-\underline{\alpha}^{2} \alpha^{2 n}-2 \underline{\alpha} \alpha^{n} \underline{\beta} \beta^{n}-\underline{\beta}^{2} \beta^{2 n}\right) .
$$

By completing the necessary abbreviations and procedures, we get

$$
B_{n+k} B_{n-k}-B_{n}^{2}=\frac{1}{5} \underline{\alpha} \underline{\beta}\left(\alpha^{n+k} \beta^{n-k}+\beta^{n+k} \alpha^{n-k}-2(-1)^{n}\right) .
$$

On the other hand, calculating the

$$
\left.\alpha^{n+k} \beta^{n-k}+\beta^{n+k} \alpha^{n-k}-2(-1)^{n}=(-1)^{n}\left\{(-1)^{k} \alpha^{k}+\beta^{k}\right)^{2}-4\right\},
$$

we find that

$$
B_{n+k} B_{n-k}-B_{n}^{2}=\frac{6}{5}(-1)^{n}(3 j-4 k)\left\{(-1)^{k}\left(\alpha^{k}+\beta^{k}\right)^{2}-4\right\} .
$$

This completes the proof.

Taking $k=1$ in Theorem 2.6, we obtain the Cassini's Identity for the bicomplex numbers $\mathbb{B C}_{C F}$.

The following theorem gives Honsberger formula involving the numbers $B_{n}$ in $\mathbb{B C}_{C F}$

Theorem 2.5 (Honsberger Formula). For the integers $n, k$ and the elements $B_{n}$ of sequence $\mathbb{B C}_{C F}$, the following formula is satisfied:

$$
B_{k-1} B_{n}+B_{k} B_{n+1}=\frac{1}{5}\left\{\underline{\alpha}^{2} \alpha^{k-1+n}\left(1+\alpha^{2}\right)+\underline{\beta}^{2} \beta^{k-1+n}\left(1+\beta^{2}\right)\right\} .
$$

Proof. Using the Binet formula for $B_{k-1} B_{n}+B_{k} B_{n+1}$ for the right-hand side of above equation, we can write

$$
\begin{aligned}
\frac{1}{\sqrt{5}}\left(\underline{\alpha} \alpha^{k-1}+\right. & \left.\underline{\beta} \beta^{k-1}\right) \frac{1}{\sqrt{5}}\left(\underline{\alpha} \alpha^{n}+\underline{\beta} \beta^{n}\right)+\frac{1}{\sqrt{5}}\left(\underline{\alpha} \alpha^{k}+\underline{\beta} \beta^{k}\right) \frac{1}{\sqrt{5}}\left(\underline{\alpha} \alpha^{n+1}+\underline{\beta} \beta^{n+1}\right) \\
& =\frac{1}{5}\left(\underline{\alpha}^{2} \alpha^{k-1+n}+\underline{\alpha} \alpha^{k-1} \underline{\beta} \beta^{n}+\underline{\beta} \beta^{k-1} \underline{\alpha} \alpha^{n}+\underline{\beta}^{2} \beta^{k-1+n}\right) \\
& +\frac{1}{5}\left(\underline{\alpha}^{2} \alpha^{k+n+1}+\underline{\alpha} \alpha^{k} \underline{\beta} \beta^{n+1}+\underline{\beta} \beta^{k} \underline{\alpha} \alpha^{n+1}+\underline{\beta}^{2} \beta^{k+n+1}\right) .
\end{aligned}
$$

So, we can now get

$$
\begin{gathered}
\frac{1}{5}\left\{\underline{\alpha}^{2}\left(\alpha^{k-1+n}+\alpha^{k+1+n}\right)+\underline{\alpha} \underline{\beta}\left(\alpha^{k-1} \beta^{n}+\beta^{k-1} \alpha^{n}+\alpha^{k} \beta^{n+1}+\beta^{k} \alpha^{n+1}\right)\right. \\
\left.+\underline{\beta}^{2}\left(\beta^{k-1+n}+\beta^{k+1+n}\right)\right\} .
\end{gathered}
$$

We can now get that

$$
\underline{\alpha} \underline{\beta}\left(\alpha^{k-1} \beta^{n}+\beta^{k-1} \alpha^{n}+\alpha^{k} \beta^{n+1}+\beta^{k} \alpha^{n+1}\right)=0 .
$$

Thus, the claim is proved. 
Theorem 2.6 (d'Ocagne's Identity). For the integers $m, n$ and the elements $B_{n}$ of sequence $\mathbb{B C}_{C F}$, we have

$$
B_{m} B_{n+1}-B_{n} B_{m+1}=\frac{6}{5}(3 j-4 k)(-1)^{m}\left(\alpha^{n-m-1}\left(\alpha^{2}+1\right)+\beta^{n-m-1}\left(\beta^{2}+1\right)\right) .
$$

Proof. Let us start by considering the right-hand side of the above equation:

$$
\begin{gathered}
=\frac{1}{\sqrt{5}}\left(\underline{\alpha} \alpha^{m}+\underline{\beta} \beta^{m}\right) \frac{1}{\sqrt{5}}\left(\underline{\alpha} \alpha^{n+1}+\underline{\beta} \beta^{n+1}\right)-\frac{1}{\sqrt{5}}\left(\underline{\alpha} \alpha^{n}+\underline{\beta} \beta^{n}\right) \frac{1}{\sqrt{5}}\left(\underline{\alpha} \alpha^{m+1}+\underline{\beta} \beta^{m+1}\right) \\
=\frac{1}{5}\left\{\left(\underline{\alpha} \alpha^{m}+\underline{\beta} \beta^{m}\right)\left(\underline{\alpha} \alpha^{n+1}+\underline{\beta} \beta^{n+1}\right)-\left(\underline{\alpha} \alpha^{n}+\underline{\beta} \beta^{n}\right)\left(\underline{\alpha} \alpha^{m+1}+\underline{\beta} \beta^{m+1}\right)\right\} .
\end{gathered}
$$

After some algebraic manipulations, we obtain

$$
B_{m} B_{n+1}-B_{n} B_{m+1}=\frac{1}{5} \underline{\alpha} \underline{\beta}\left(\alpha^{m} \beta^{n+1}+\beta^{m} \alpha^{n+1}-\alpha^{n} \beta^{m+1}-\beta^{n} \alpha^{m+1}\right) .
$$

Calculating the following equality, we have

$$
\alpha^{m} \beta^{n+1}+\beta^{m} \alpha^{n+1}-\alpha^{n} \beta^{m+1}-\beta^{n} \alpha^{m+1}=(-1)^{m} \alpha^{n-m-1}\left(\alpha^{2}+1\right)+\beta^{n-m-1}\left(\beta^{2}+1\right) \text {. }
$$

Thus, we get

$$
B_{m} B_{n+1}-B_{n} B_{m+1}=\frac{6}{5}(3 j-4 k)\left((-1)^{m} \alpha^{n-m-1}\left(\alpha^{2}+1\right)+\beta^{n-m-1}\left(\beta^{2}+1\right)\right) .
$$

Thus, the proof is completed.

Theorem 2.7 (Vajda's Identity). For the integers $m, n, k$ and the number $B_{n}$, we have

$$
B_{n+m} B_{n+k}-B_{n} B_{n+m+k}=\frac{6(-1)^{n}}{5}(3 j-4 k)\left(\alpha^{k}-\beta^{k}\right)\left(\beta^{m}-\alpha^{m}\right) .
$$

Proof. The formula

$$
B_{n+m} B_{n+k}-B_{n} B_{n+m+k}
$$

is equal to this:

$$
\left.\frac{1}{5}\left\{\left(\underline{\alpha} \alpha^{n+m}+\underline{\beta} \beta^{n+m}\right)\left(\underline{\alpha} \alpha^{n+k}+\underline{\beta} \beta^{n+k}\right)-\left(\underline{\alpha} \alpha^{n}+\underline{\beta} \beta^{n}\right) \underline{\alpha} \alpha^{n+m+k}+\underline{\beta} \beta^{n+m+k}\right)\right\} .
$$

If we assume that some arrangements and arithmetic operations are performed, then we get:

$$
\begin{aligned}
B_{n+m} B_{n+k}-B_{n} B_{n+m+k} & =\frac{(-1)^{n}}{5} \underline{\alpha} \underline{\beta}\left\{\alpha^{m}\left(\beta^{k}-\alpha^{k}\right)+\beta^{m}\left(\alpha^{k}-\beta^{k}\right)\right\}, \\
B_{n+m} B_{n+k}-B_{n} B_{n+m+k} & =\frac{6(-1)^{n}}{5}(3 j-4 k)\left(\alpha^{k}-\beta^{k}\right)\left(\beta^{m}-\alpha^{m}\right) .
\end{aligned}
$$

Thus, the correctness of the claim is seen. 
Theorem 2.8 (Gelin-Cesaro Identity). For the numbers $B_{n}, n \geq 2$, the following identity is satisfied:

$$
B_{n-2} B_{n-1} B_{n+1} B_{n+2}-B_{n}^{4}=\frac{3}{50} \underline{\alpha} \underline{\beta}\left\{(-1)^{n}\left(\underline{\alpha}^{2} \alpha^{2 n}+\underline{\beta}^{2} \beta^{2 n}\right)-15\right\} .
$$

Proof. Let us start by considering the right-hand side of equation

$$
B_{n-2} B_{n-1} B_{n+1} B_{n+2}-B_{n}^{4}
$$

and use the formula

$$
B_{n}=\frac{1}{\sqrt{5}}\left\{\left(B_{1}-\beta B_{0}\right) \alpha^{n}+\left(\alpha B_{0}-B_{1}\right) \beta^{n}\right\}
$$

Then, we have

$$
\begin{aligned}
& \frac{1}{25}\left\{\left(\underline{\alpha} \alpha^{n-2}+\underline{\beta} \beta^{n-2}\right)\left(\underline{\alpha} \alpha^{n-1}+\underline{\beta} \beta^{n-1}\right)\left(\underline{\alpha} \alpha^{n+1}+\underline{\beta} \beta^{n+1}\right)\left(\underline{\alpha} \alpha^{n+2}+\underline{\beta} \beta^{n+2}\right)\right\} \\
& \quad-\frac{1}{25}\left\{\left(\underline{\alpha} \alpha^{n}+\underline{\beta} \beta^{n}\right)^{4}\right\} \\
& =\frac{1}{25}\left(\underline{\alpha}^{3} \underline{\beta} \alpha^{3 n} \beta^{n}\left(\alpha^{-1} \beta+\alpha \beta^{-1}+\alpha^{2} \beta^{-2}+\alpha^{-2} \beta^{2}-4\right)+\underline{\alpha}^{2} \underline{\beta}^{2}\left(\alpha \beta^{-1}+\alpha^{3} \beta^{-3}\right.\right. \\
& \left.\left.\quad+\alpha^{-3} \beta^{3}+\alpha^{-1} \beta-4\right)+\underline{\alpha} \underline{\beta}^{3} \alpha^{n} \beta^{3 n}\left(\alpha^{2} \beta^{-2}+\alpha^{-2} \beta^{2}+\alpha^{-1} \beta+\alpha \beta^{-1}-4\right)\right) .
\end{aligned}
$$

Making the necessary arrangements, we obtain that $B_{n-2} B_{n-1} B_{n+1} B_{n+2}-B_{n}^{4}$ is equal to

$$
\frac{3}{50} \underline{\alpha} \underline{\beta}\left\{(-1)^{n}\left(\underline{\alpha}^{2} \alpha^{2 n}+\underline{\beta}^{2} \beta^{2 n}\right)-15\right\} \text {. }
$$

Thus, the claim is true.

\section{Conclusion}

In this work, we defined a new sequence of bicomplex numbers. The elements of this sequence have the coefficients from complex Fibonacci numbers. We examined some important properties of these newly defined numbers and gave their Binet formula. By the help of the Binet formula, we also obtained the respective generalizations of some well-known important identities involving these numbers.

\section{Acknowledgements}

The authors would like to thank the anonymous reviewers for their useful comments and suggestions.

\section{References}

[1] Anastassiu, H. T., Atlamazoglou, P. E., \& Kaklamani, D. I. (2003). Application of bicomplex (quaternion) algebra to fundamental electromagnetics: A lower order alternative to the Helmholtz equation, IEEE Transactions on Antennas and Propagation, 51 (8), 2130-2136. 
[2] Deveci, O., \& Shannon, A. G. (2018). The quaternion-Pell sequence, Communications in Algebra, 46 (12), 5403-5409.

[3] Deveci, O., \& Shannon, A. G. (2018). The Redheffer numbers and their applications Notes on number theory and discrete math., 4 (4), 26-37.

[4] Flaut, C., \& Savin, D. (2014). About quaternion algebras and symbol algebras Bulletin of the Transilvania University of Brasov. Mathematics, Informatics, Physics., Series III, 7 (2), 59.

[5] Halıc1, S. (2012). On fibonacci quaternions Advances in Applied Clifford Algebras, 22 (2), 321-327.

[6] Halıc1, S. (2013). On complex Fibonacci quaternions. Advances in Applied Clifford Algebras, 23 (1), 105-112.

[7] Halıc1, S. (2019). On Bicomplex Fibonacci Numbers and Their Generalization. In: Models and Theories in Social Systems, Springer, Cham, 509-524.

[8] Hamilton, W. R. (1853). Lectures on Quaternions: Containing a Systematic Statement of a New Mathematical Method; of which the Principles Were Communicated in 1843 to the Royal Irish Academy; and which Has Since Formed the Subject of Successive Courses of Lectures, Delivered in 1848 and Sub Sequent Years, in the Halls of Trinity College, Dublin: Withnumerous Illustrative Diagrams, and with Some Geometrical and Physical Applications. University Press by $\mathrm{MH}$.

[9] Horadam, A. F. (1963). Complex Fibonacci numbers and Fibonacci quaternions, The American Mathematical Monthly, 70 (3), 289-291.

[10] Horadam, A. F. (1993). Quaternion recurrence relations Ulam Quarterly, 2 (2), 23-33.

[11] Kabadayi, H., \& Yayli, Y. (2011). Homothetic motions at $E^{4}$ with bicomplex numbers, Advances in applied Clifford algebras , 21 (3), 541-546.

[12] Koshy, T. (2001). Fibonacci and Lucas numbers with applications. Vol. 1. John Wiley, Sons.

[13] Luna-Elizarraras, M. E., Shapiro, M., Daniele, C. S., \& Vajiac, A. (2012). Bicomplex numbers and their elementary functions, Сubo (Тетисо), 14 (2), 61-80.

[14] Luna-Elizarraras, M. E., Shapiro, M., Daniele, C. S., \& Vajiac, A. (2015). Bicomplex holomorphic functions, The Algebra, Geometry and Analysis of Bicomplex Numbers. Birkhauser.

[15] Nurkan Kaya, S., \& Guven, I. (2015). A Note On Bicomplex Fibonacci and Lucas Numbers, arXiv preprint arXiv:1508.03972.

[16] Rochon, D., \& Shapiro, M. (2004). On algebraic properties of bicomplex and hyperbolic numbers, Anal. Univ. Oradea, Fasc. Math, 11 (71), 110. 
[17] Rochon, D., \& Tremblay, S. (2004). Bicomplex quantum mechanics: I. The generalized Schrodinger equation, Advances in Applied Clifford Algebras, 14 (2), 231-248.

[18] Segre, C. (1892). Le rappresentazioni reali delle forme complesse a gli enti iperalgebrici Mathematische Annalen, 40, 413-467. 